\title{
Role of PmRab7 Regulation in WSSV Infection and Functional Validation of Small Molecule as PmRab7 GTPase Inhibitor
}

\author{
Amrendra Kumar, Sudhanshu Shekhar and Saravanakumar A* \\ Department of Marine Sciences, Centre of Advanced Study in Marine Biology, Annamalai University, Chidambaram, India
}

\begin{abstract}
White Spot Syndrome (WSS) is a viral disease has extensive threatened the shrimp farming industries. It is caused by White spot syndrome baculovirus (WSSB). The shrimps (P. monodon and L. vannamei) are unable to defend themselves by "nonself" materials and pathogen, due to less define adaptive immune response. Therefore, the present work was taken on priority to study the regulation of PmRab7 transcript, which might be a receptor for WSSV (vP28) after implication of small molecule inhibitor. Total 70 GTPase small molecules were screened initially, against the 3D structure of PmRab7. Among all, the CID 1067700 molecule was selected for molecular docking (IFD) with PmRab7 in presence of GTP, GDP and $\mathrm{Mg}^{++}$. Furthermore, a small molecule was fed in feed formulation to $P$. monodon and transcriptional regulation of $P m R a b 7$ was relatively quantified against IFN- $\alpha$ as housekeeping genes during the WSSV infection. Herein we identified a PmRab7 inhibitor, CID 1067700, by targeting DNA binding domain of PmRab7 using virtual screening strategy. CID 1067700 preferentially suppresses GTPase activity to alter GTP and $\mathrm{Mg}^{++}$from their positions. Furthermore, CID 1067700 inhibits expression of PmRab7 downstream target genes thus CID 1067700 represent novel probes for the development of specific inhibitors targeting DNA binding domain of PmRab7 and a potential therapeutic against WSSV. Substantial changes were observed in PmRab7 regulation and WSSV multiplication in the initial trial of CID 106700. CID 106700 leads towards successful inactivation of PmRab7 by altering the GTP and $\mathrm{Mg}^{++}$from their native positions. The PmRab7 regulation was increased 5 to 8 -fold during 72 hrs of infection. GTP replacement by small molecules has revealed significantly down regulation of PmRab7 gene during WSSV infection. Remarkably, the viral load (absolutely quantified) also has been found to be reduced when the PmRab7 was down-regulated. WSSV internalization has directly linked with regulation of PmRab7.
\end{abstract}

Keywords: P. monodon; WSSV; PmRab7; GTPase; Inhibitor; Gene regulation

\section{Introduction}

PmRab7 is Ras-related (Rat Sarcoma) protein found in Penaeus monodon (an important seafood species). It is encoded by 205 amino acid contain five conserved motifs which consist five GTPbinding sites (G-BOX), two switch regions, GTPase activity, and an isoprenylation site. PmRab7 directly involved in binding with white spot syndrome viruses (WSSV) during infection which causes huge mortality and loss billions of dollars. More than 70 Rab isoforms have been identified in mammals but the function of each isoform is poorly understood but among them, Rab1, Rab5, Rab6, Rab7, and Rab11 are known as housekeeping genes which are conserved from yeast to humans [1,2]. The function of small GTPase Rab7 is regulation of motility, promoting plus-end-directed transport of autophagosomes, recruitment of the retromer to the LE (Late endosome) membrane and endosomal maturation process are some major functions of Rab7 [3]. Membrane association of Rabs is mediated by a geranyl-geranyl group which is added post-translationally to cysteine residues present at the C-terminus. The C-terminal "hypervariable" region of Rabs is the least conserved region across members of the Rab family. Inactive Rabs are largely sequestered in the cytosol by Rab GDP-dissociation inhibitor (GDI) which recognizes GDP-bound Rabs and binds their C-terminal prenyl group $[4,5]$. Protecting the hydrophobic C-terminal lipids of Rabs, GDI also inhibits the dissociation of GDP from Rabs by inducing a conformational change that sterically blocks the opening to the nucleotide binding pocket [6]. GDI is also important for delivery of inactive, GDP-bound Rabs to the appropriate membrane compartment $[7,8]$. The function of Rab7 is similar to other Rabs by recruitments of the specific regulator at upstream and effectors at a downstream part to the membrane during GTP-bound active state [9-14]. Intrinsic GTP hydrolysis rates of Rabs are characteristically low, so efficient hydrolysis requires for the activity of a GTPase activating protein (GAP). Like other effector proteins, GAPs specifically recognize the GTP-bound state conformation of Rab7 and other Rabs [15]. Although the glutamine residue of the DxxGQ (Q67 in Rab7) is generally considered to be catalytic in GTP hydrolysis, recent evidence suggests that this might not be the case in Rab GTPases that bind TBC family members. Remarkably, most Rab GTPases shows intrinsically low rates of GDP dissociation and GTP hydrolysis. Because of these properties, both the spontaneous rates of activation and inactivation of Rab GTPases are usually quite slow and allow the Rab activity cycle to be under positive and negative modulatory influences of GEF and GAP proteins [16,17]. Here, we report a smallmolecule inhibitor of PmRab7 GTPase targeting PmRab7 activation by Guanine Exchange Factors (GEF). The chemical compound CID 1067700 was identified by a structure-based virtual screening which fit into a groove of PmRab7 known to be critical for GEF specification. The small chemical molecule 2-(benzoylcarbamothioylamino)-5,5dimethyl-4,7-dihydrothieno[2,3-c] pyran-3-carboxylic acid (PubChem CID 1067700) as an inhibitor of nucleotide binding by Ras related GTPases. The mechanism of action of these PmRab7 GTPase inhibitors is not well characterized, in the context of other Rab7 GTPase as there are no known inhibitors of Rab GTPases. The compound behaves as

*Corresponding author: Saravanakumar A, Department of Marine Sciences, Centre of Advanced Study in Marine Biology, Annamalai University, Chidambaram, India, Tel: 04144238 282; E-mail: asarvaan@gmail.com

Received April 06, 2017; Accepted October 23, 2017; Published October 25, 2017

Citation: Kumar A, Shekhar S, Saravana kumar A (2017) Role of PmRab7 Regulation in WSSV Infection and Functional Validation of Small Molecule as PmRab7 GTPase Inhibitor. J Aquac Res Development 8: 511. doi: 10.4172/21559546.1000511

Copyright: ( 2017 Kumar A, et al. This is an open-access article distributed under the terms of the Creative Commons Attribution License, which permits unrestricted use, distribution, and reproduction in any medium, provided the original author and source are credited. 
a competitive inhibitor of Rab7 nucleotide binding based on both equilibrium binding and dissociation assays. Hence the present study the, structural and functional properties of $P m R a b 7$, involvement of GTPase as a key regulator of PmRab7 either in GTP-bound condition or GDP-bound state condition, screening of small molecule as PmRab7 GTPase inhibitor to make functionally unable to PmRab7 by using different pose, binding site and interaction points to make conformational change in PmRab7.

\section{Materials and Methods}

\section{DNA sequencing molecular docking}

Sequence was submitted to NCBI Genebank (KF199870.1), 3D model structure was generated by PyMOL with the reference to $1 \mathrm{vg} 8$ (PDB protein ID) on PDB. The structure was docked by Patchdoc and Glydoc (commercially available) for fit binding of $\mathrm{Mg}^{2+}$ and GTP. Schrodinger suite was used to prepare protein, the proteins were pre-processed separately by deleting the substrate-cofactor as well as the crystallographically observed water molecules (water without $\mathrm{H}$ bonds) optimizing hydrogen bonds. LigPrep module was used for ligand preparation by using the LigPrep module; the drawn ligands were geometry optimized by using the optimized potentials for liquid simulations-2005 (OPLS-2005) force field with the steepest descent followed by truncated Newton Conjugate gradient protocol, further structure was used for receptor grid generation, the ligands were rigid docked with the protein by using module in extra precision mode (XP). Induced fit docking was performed to achieve deeper residence of the ligand within the cavity.

\section{Docking accuracy}

The performance of the docking program was evaluated by comparing the docked pose of ligands to co-crystallize ligand pose in the 1W51 crystal structure. The interactions were viewed using Ligplot and PyMOL.

\section{Experimental animal and CID 1067700 administration}

Shrimps ( $P$. monodon) weighing $20 \pm 2 \mathrm{~g}$ were collected from Vellar estuary (Lat $11^{0} 30^{\prime} 16.94^{\prime \prime} \mathrm{N} ; 79^{\circ} 46^{\prime} 39.53^{\prime \prime}$ E), South east cost of India, were stocked and reared in a RAS for shrimp integrated with nitrifying bioreactors where; pH-7.6 to 8.25, Salinity about $15 \mathrm{ppt}$, Ammonia $<0.001$ to $0.2 \mathrm{ppm}, \mathrm{NO}_{2} 0.1$ to $0.3 \mathrm{ppm}$ and dissolved oxygen $5-6 \mathrm{mg}$ $\mathrm{L}^{-1}$ was maintained. The shrimp were fed with commercial pelleted feed (Higashimaru, India) and maintained for acclimatized. Two different sets of treatments were considered for sampling. Calculated quantity of appropriate feed type was commercial pelleted feed (Higashimaru, India) and CID 1067700 coated feed.

\section{WSSV challenge}

The quantity $\left(10^{-3}\right.$ copies/ $\left.\mu \mathrm{l}\right)$ of WSSV infected tissue was taken for oral challenge (lowest quantity of WSSV infected head soft tissues required for causing $100 \%$ mortality in P. monodon within 7 days of administration). The experimental animals were challenged with WSSV infected head soft tissues (at $2 \%$ of body weight of experimental animals) on $1^{\text {st }}$ day, $5^{\text {th }}$ day and $10^{\text {th }}$ day post- administration of the appropriate feed type.

\section{Collection of samples and storage for analysis}

The samples were collected on $1^{\text {st }}$ day, $5^{\text {th }}$ day and $10^{\text {th }}$ day after 7 days of administration of the appropriate feed type (small molecule coated/ Normal) as control to the challenged set of animals. Before sampling animals were anesthetized, dissection was carried out in sterile condition and snap freeze immediately. Samples were immediately stored in $\mathrm{TRI}^{\ominus}$ Reagent (Sigma) at $-80^{\circ} \mathrm{C}$ for RNA extraction. Gills and the haemocyte lysate were also stored in $-80^{\circ} \mathrm{C}$ for protein profiling. Pleopods for PCR were fixed in $70 \%$ ethanol and stored in $4^{\circ} \mathrm{C}$. The experimental animals were also fixed by injecting and immersing in Davidson's fixative for histopathology. The detailed procedures for the collection and treatment of samples for each of the above-mentioned analysis have been dealing with in the respective areas in the following methods.

\section{Total RNA extraction and cDNA synthesis}

The total RNA was extracted using TRIzol reagent (Invitrogen, USA) by following the manufacturer's protocol, purity was measured by NanoDrop and RNA quality was calculated by $1 \%$ gel electrophoresis. cDNA was synthesized from the RNA using the First strand cDNA synthesizing kit (Fermentas, USA) by using Moloney Murine leukemia virus reverse transcriptase (M-MuLV) following the manufacturer's protocol with help of RT-PCR.

\section{WSSV copy number study and transcript distribution study using qRT-PCR}

Viral copy number in experimental samples determined by quantitative real-time PCR using Maxima TH SYBR Green qPCR master mix reagent (Fermentas and Applied Biosystem USA). The standard curve was first created. Eight serial dilutions of WSSV plasmids of known copy numbers $\left(10^{8}, 10^{7}, 10^{6}, 10^{5}, 10^{4}, 10^{3}, 10^{2}, 10^{1}, 10^{0}\right.$ copies/ $\mu \mathrm{l}$ ) were prepared and used as templates for RT-PCR reactions. A standard curve was made by plotting plasmid copy numbers against their respective mean $\mathrm{Ct}$ values. For expression of PmRab7 mRNA level, the cDNA concentrations of all samples were adjusted to 100 ng/ $\mu \mathrm{L}$. Relative mRNA expression of $P m R a b 7$ by forward and reverse primer was measured by qRT-PCR using interferon (IF)- $1 \alpha$ primers as the internal standard (housekeeping gene). The amplifications were performed in a 96-well plate. The qRT-PCR mixture contained 1.25 $\mu \mathrm{l}$ of cDNA, $10 \mu \mathrm{l}$ of nuclease free water, $12.5 \mu \mathrm{l}$ of $2 \mathrm{X}$ SYBR Green PCR master mixes and $0.625 \mu \mathrm{l}$ of each gene specific primer (stock concentration of $0.25 \mu \mathrm{M}$ ). The amplification programme consisted of $95^{\circ} \mathrm{C}$ for $10 \mathrm{~min}$ followed by 40 cycles of $95^{\circ} \mathrm{C}$ for $15 \mathrm{sec}, 60^{\circ} \mathrm{C}$ for 1 min. All reactions were performed in duplicates (each well containing $10 \mu$ l of master mix).

\section{Ethics statement}

The guidelines of the Committee for the Purpose of Control and Supervision of Experiments on Animals, Ministry of Environment and Forests, Govt of India were followed during experiment. Methods used in this study were approved by expert committee of Annamalai University.

\section{Statistical analysis}

One-way ANOVA performed to fit the distribution normal natural log of each copy number of WSSV, the correlation coefficient was determined to plot the log value of copy numbers of WSSV. The differences in PmRab7 transcript level between different time points were tested for statistical significance using the statistical package SPSS 16.0 (USA). Data was subjected to one-way analysis of variance (ANOVA), followed by Duncan's Multiple Range Test. $\mathrm{P}<0.05$ was considered as statistically significant. The $2^{-\Delta \Delta C T}$ method [18] was used to analyze the expression level of PmRab7. 


\section{Results}

\section{In-silico study}

Our previous study showed that identities of PmRab7 proteins are 67\% (PgRab7), 86\% (HsRab7 and RnRab7), and LvRab7 was 99\% respectively, based on public databases search, sequence annotation and similarity enabled us together as many as 61 non-redundant sequences representing Rab7 proteins. The search showed that Rab7 proteins were represented in all supergroups of RAS related proteins. Phylogenetic relationships between all Rab7 and Rabs proteins constructed based on their amino acid sequences, Cascade of Rabs protein arranged at single step from 0.550 to 0.950 where as other involved effector proteins and other GTPas shows long distance (1.000) (Figure 1). These sequences cluster together with a strong bootstrap support and clearly separate from representatives of the closest Rab subfamilies 6,17, 21,23, 29, 32, 38 etc from Rab family, similarly from Ran, Rac, Arf, Ras and Rho family with various regulator and effectors.

\section{D model structure prediction and GTP binding site}

Given sequence structure in PDB shows $90 \%$ similar to PDB ID 1Vg8, furthermore the supplemented $\mathrm{Mg}^{2+}$, GDP and GTP. The different protein properties were showed in $P m R a b 7$ sequence like $\mathrm{GTP} / \mathrm{Mg}^{2+}$ has been found in between 17 and 157 amino acid at different four places, GDI interaction site were found from 17 to 79 amino acid, PmRab7 conserve domain conation five G box, two molecular switch regions, five Rab subfamily motif and two putative GEF interaction sites (Figures $2 \mathrm{a}$ and $2 \mathrm{~b}$ ).

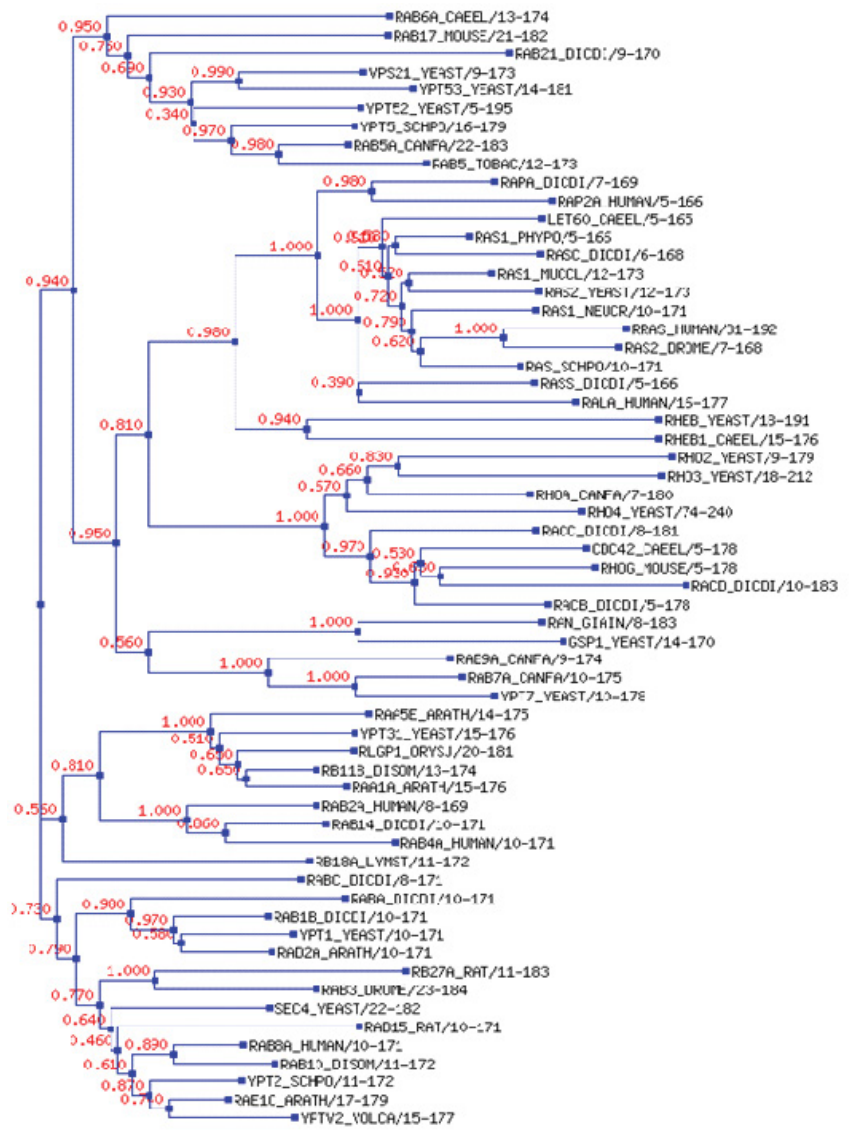

Figure 1: Distance relation between different RabGTPas protein family in under Ras related super family.

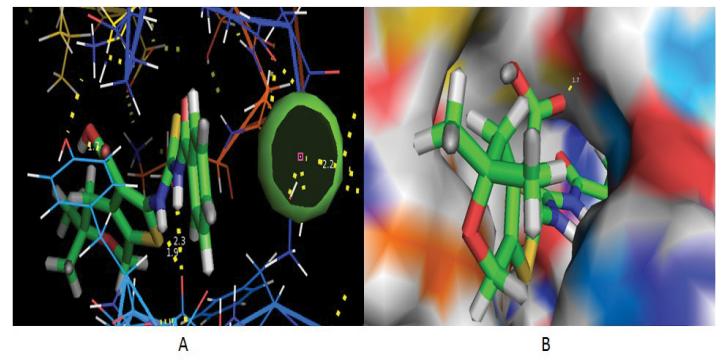

Figure 2 (a): Interaction of GTP with PmRab7 in presence of Mg2+ and (b) GTP binding pocket of PmRab7.

\begin{tabular}{|c|c|c|}
\hline Compound & Docking score & Glide energy (Kcal/mol) \\
\hline CID_1067700 & 7.89 & -73.55 \\
\hline CID_740871 & 2.32 & -56.45 \\
\hline CID_817029 & 2.17 & -55.71 \\
\hline CID_1067699 & 1.88 & -48.01 \\
\hline CID_1021465 & 1.78 & -47.26 \\
\hline CID_898979 & 1.37 & -45.21 \\
\hline CID_673347 & 1.02 & -44.90 \\
\hline CID_1068266 & 0.91 & -43.11 \\
\hline CID_1097649 & 0.57 & -41.19 \\
\hline CID_1068267 & -1.09 & -36.20 \\
\hline
\end{tabular}

Table 1: Screening of high scoring compounds.

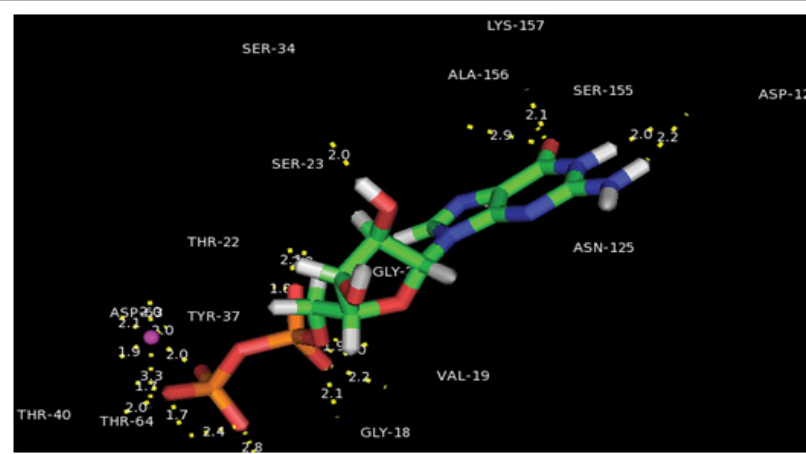

Figure 3: Interaction of GTP, $\mathrm{Mg}^{2+}$ and $P m R a b 7$ amino acid residues.

\section{Molecular docking}

CID 1067700 as a GTPase inhibitor was attached on define binding site in induce fit docking by Glydoc. Best $\mathrm{Mg}^{2+}$ and GTP binding sites were chosen on high score and high energy value. After screening of the PubChem library compounds, the scores were sorted based on High Throughput Virtual Screening (HTVS). In primary screening, 10 compounds were selected out of 70. The GLIDE docking method is applied to inhibitors to build an affinity model with the PmRab7 of different inhibitors generated by high scoring facility. It was characterized by orientations and hydrogen bond positions. These observations show that inhibition of the ligand is depend on the various conformations with the rigid type of docking (Table 1). The CID 1067700 shows a best interaction with SER and TYR, of PmRab7 whereas GTP shows best interaction with ALA, SER, ASP, THR, TYR and THR during flexible docking (Figures 3 and 4). Ligands were filtered by using high throughput virtual screening approaching using GLIDE docking, in which the high energy molecules were taken. During the docking process the ligands were rigid and receptor treated as rigid. Furthermore, the ligands were docked into GTP binding sites 


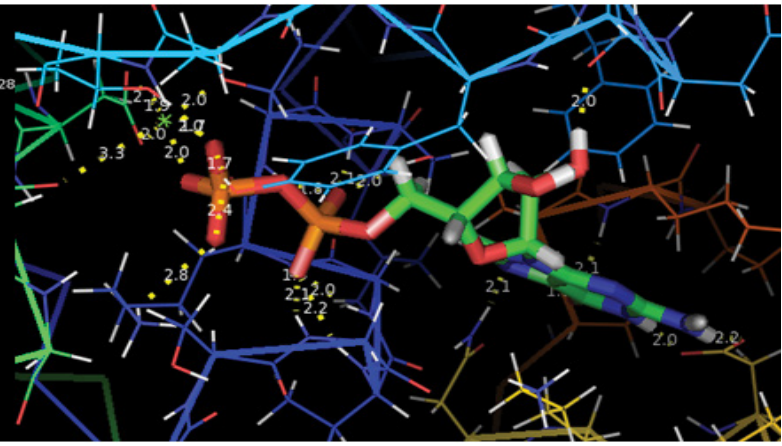

Figure 4: Interaction of GTP, $\mathrm{Mg}^{2+}$ and PmRab7 amino acid residues atoms PmRab7 amino acid residues involved in binding of GTP and magnesium.

\begin{tabular}{|c|c|c|c|c|}
\hline $\begin{array}{l}\text { Compound } \\
\text { name }\end{array}$ & $\begin{array}{l}\text { Docking } \\
\text { score }\end{array}$ & $\begin{array}{l}\text { Glide energy } \\
\text { (Kcal/mol) }\end{array}$ & $\begin{array}{c}\text { Interaction } \\
\text { D-H...A }\end{array}$ & $\begin{array}{c}\text { Distance } \\
(\hat{A})\end{array}$ \\
\hline \multirow{6}{*}{ GTP } & \multirow{6}{*}{9.22} & \multirow{6}{*}{-62.41} & $\begin{array}{l}\mathrm{N}-\mathrm{H} \ldots \mathrm{O} \\
(\mathrm{ALA156})\end{array}$ & 2.1 \\
\hline & & & $\mathrm{N}-\mathrm{H}$... O (SER34) & 2.9 \\
\hline & & & $\begin{array}{l}\mathrm{N}-\mathrm{H} \ldots \mathrm{O} \\
(\mathrm{ASP} 128)\end{array}$ & $2.0-2.2$ \\
\hline & & & $\mathrm{N}-\mathrm{H} . . . \mathrm{O}(\mathrm{THR} 22)$ & 2.1 \\
\hline & & & $\mathrm{N}-\mathrm{H} \ldots \mathrm{O}$ (TYR37) & 1.6 \\
\hline & & & PHO...O(THR64) & 2 \\
\hline \multirow{3}{*}{ CID-1067700 } & \multirow{3}{*}{7.89} & \multirow{3}{*}{-73.55} & $\mathrm{~N}-\mathrm{H} . . . \mathrm{O}$ (SER34) & 2.3 \\
\hline & & & $\mathrm{N}-\mathrm{H} . . . \mathrm{O}$ (SER34) & 1.9 \\
\hline & & & N-H... O (TYR37) & 1.7 \\
\hline
\end{tabular}

Table 2: Comparative virtual screening results of GTP and CID-1067700.

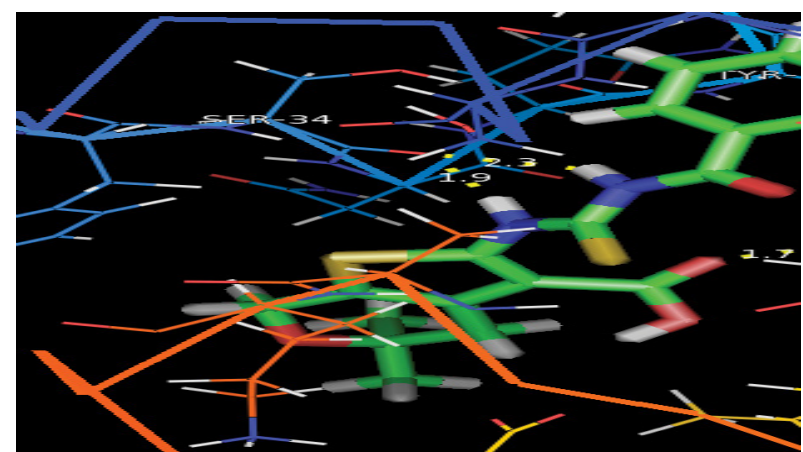

Figure 5: Biding of CID_1067700 small molecule with PmRab7 amino acid residues after replacing GTP by heating $\mathrm{Mg}^{2+}$.

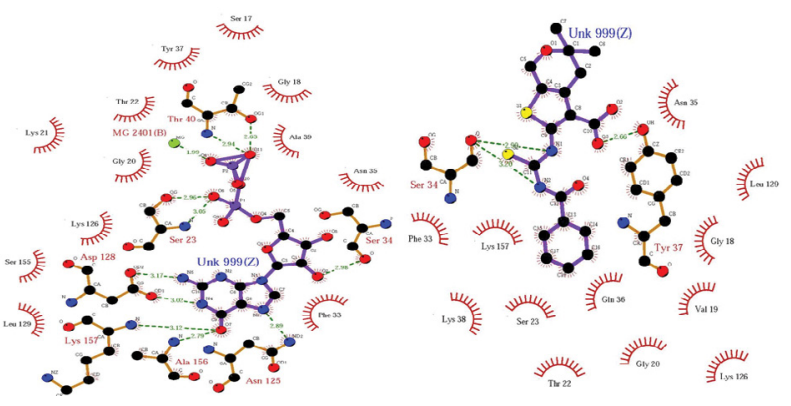

Figure 6: Protein-ligand interaction using Ligplot, which shows the better interaction with aspartate, threonine, arginine and cofactor $\mathrm{Mg}^{2+}$.

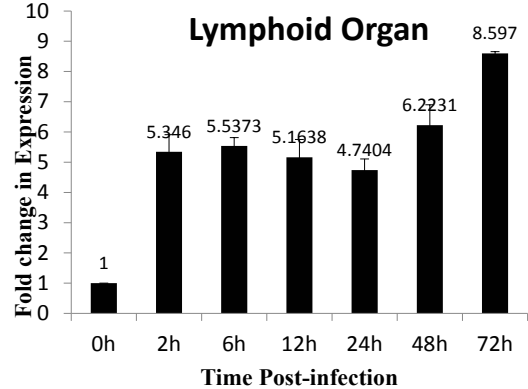

Figure 7: PmRab7 gene differential expression from 0 to $72 \mathrm{hrs}$ of WSSV infection.

of the $P m R a b 7$ during induce fit docking, where the $P m R a b 7$ may rigid and the ligand was free to move, that has been filtered out from many compounds using virtual screening. The PmRab7 shows the critical hinging and displays more conformations, the conformations show that many alterations in the PmRab7 GTPase and it is more closely conforms and fit to the ligands moreover it shows the binding between the ligands into a rigid PmRab7, assumed by low energy value. The GTP was shown $-62.41 \mathrm{Kcal}$ whereas inhibitor compound shown CID $1067700-73.55 \mathrm{Kcal}$ as least energy (Table 2). In case of GTP interaction when $P m R a b 7$ rotation started the interaction of ALA156, SER34, ASP128, THR22, TYR37 and THR64, whereas CID 1067700 SER34 and TYR37can be noticed with Phosphate and other side chain atom (Figure 5). An auto-phosphorylation taken place in the regions of SER135, THR154 AND TYR151 the regions of PmRab7, SER were found in active site residues which shows binding towards the ligand. Further it shows the activity of protein substrates and GTP as well as having an option of placing the residues critical for Mg-GTP binding its catalysis.

\section{Ligplot analysis}

The analysis shows the pattern of interactions protein-ligand complexes and other metal ions (Figure 6), where it binds, allowing a fast analysis of the location of specific intermolecular interactions with respect to the sequence. This analysis showed the better interaction between the ligand and macromolecule. It represents the intermolecular interactions and their strengths, including hydrogen bonds, hydrophobic interactions and atom accessibilities.

\section{Effect of inhibitor on the copy number of WSSV and expres- sion patern of $P m R a b 7$}

According to the nucleotide sequence of $P m R a b 7$ in NCBI Genbank, specific primer was designed by using Applied Biosystem software for PmRab7. The expression profile of $P m R a b 7$ was detected in lymphoid tissue of $P$. monodon by Real time PCR against WSSV infection after different time points which clearly indicating the WSSV internalization of WSSV in lymphoid organ. Our previous study showed significant change in $P m R a b 7$ expression among different time point of infection. The expression of PmRab7 mRNA was at lowest level of PmRab7 mRNA was in 2 hours of infection and higher in 72 hours of infection. Significantly all-time point has shown up-regulation of $P m R a b 7$ after infection, minimum in 2-fold increases and significantly increased up to 8 -fold in $72 \mathrm{hrs}$ infection (Figure 7) against the IFN1 transcript as endogenous reference (housekeeping) gene transcript, IFN1 mRNA expression level was does not show changes relative to PmRab7. Where $P m R a b 7$ mRNA transcript expression level increased when specimens 


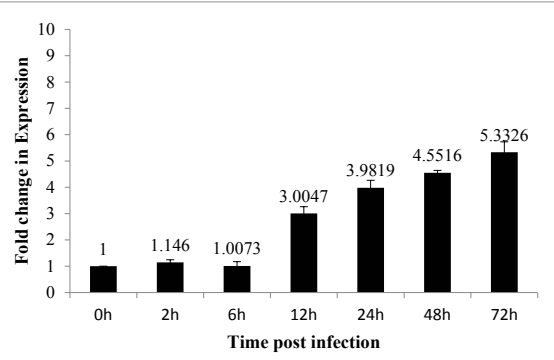

Figure 8: Differential gene expression profiling of PmRab7 upto 72 hrs of WSSV infection after use of small molecule PmRab7 GTPase inhibitor.

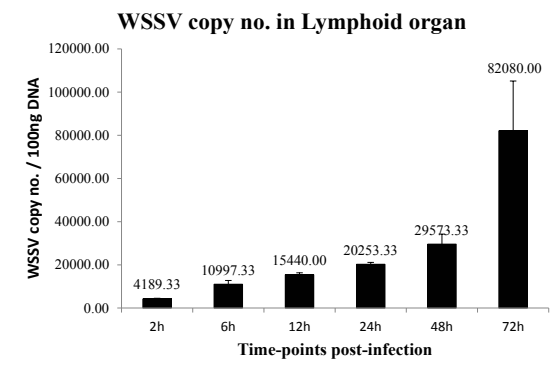

Figure 9: WSSV copy number in P. monodon after 2 to 72 hrs of infection.

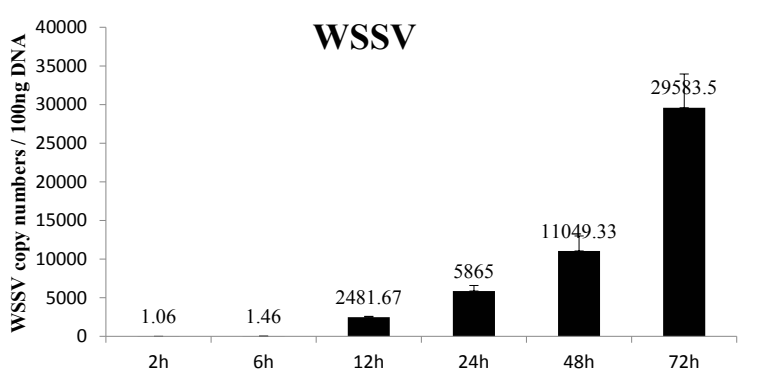

Figure 10: WSSV copy number in $P$. monodon after WSSV challenge from 2 to 72 hrs after functional inhibition of PmRab7 transcript by using PmRab7 GTPase inhibitor.

were infected with WSSV $\Delta \Delta \mathrm{Ct}=1.0$ to $\Delta \Delta \mathrm{Ct}=8.5$. This result strongly supports that WSSV enable to induce expression of $P m R a b 7$ in $P$. monodon with increasing WSSV infection at time point simultaneously increase expression of PmRab7. Whereas the effect of $P m R a b 7$ GTPase inhibitor can also see on mRNA transcript level of PmRab7, after implication of small molecule inhibitor differential expression of PmRab7 mRNA transcript has shown confined expression (Figure 8), up to 2-fold increased after $72 \mathrm{hrs}$ of infection from $\Delta \Delta \mathrm{Ct}=1.0$ to $\Delta \Delta \mathrm{Ct}=2.5$.

\section{WSSV copy number in lymphoid organ after infection}

The standard curve was generated from samples of purified cloned WSSV plasmid ranging from $2.4 \times 10^{-3}$ copies. Copy numbers were calculated by interruption of the experimentally determined threshold cycle (CT) as previously described for IHHNV by Tang and Lightner [19]. The results were expressed as the mean copy number of WSSV per microgram of total DNA. Quantification and copy number determination of WSSV using real-time PCR, WSSV infection of lymphoid cells at different time-points. The viral load in WSSV showed more than fivefold increase at $24 \mathrm{~h}$ post-infection in compared to 2 $\mathrm{h}$ post-infection and more that 20 -fold high at 72 hours of infection when exposed to a diluted WSSV inoculums 1:10 (Figure 9). Effect of CID 1067700 small molecule as PmRab7 GTPase inhibitor in WSSV internalization was clearly shown by copy number determination of WSSV in treated group of specimens. After treatment of small molecular inhibitor, differential determination of viral copy number could see clearly according to per hours viral load in compare to normal WSSV internalization, without inhibitor WSSV has shown 3 to 4-fold viral load enhanced after 2-6 hrs of infection, whereas in small molecule treated specimens does not shown viral copy in 2-6 hrs of infection, but after 6 hours viral multiplication was high (Figure 10).

\section{Discussion}

Although white spot syndrome remains a serious global problem in sea food production (shrimp) due to high mortality and less define immune system, till the date several groups are working to develop a potential drug (herbal, antibody, gene silencing and suppression of receptor gene (PmRab7) expression by dsRNA [20]. There is concurrent agreement that new antiviral drug is needed to shorten or simplify treatment by focusing the structural orientation, active site and week interaction of amino acid of PmRab7. The Rab7 domain of PmRab7 from P. monodon has one highly conserve region, block, five G box, two switch regions and one GTP binding site similar to all Rab7 monomeric from plant to animal, with one G box and GTP binding rich amino acids that will participate to bind up with $\mathrm{Mg}^{2+}$ and GTP [21]. In agreement with this, the PmRab7 gene sequence codes for a deduced polypeptide containing five extremely conserved motifs ( $G$ boxes) involved in GTP-binding or GTPase activity and an isoprenylation site (last both C), suggesting that PmRab7 is an active GTPase that is able to cycle between GDP- and GTP bound states [22]. In this study we presented 3D model of $P m R a b 7$ for $\mathrm{Mg}^{2+}$ and GTP binding site analysis, the same model was shown by Arunima et al. [23] also, but here first time we are reporting the inactive $P m R a b 7$ (GDP) to active PmRab7 (GTP) on the basis of various hydrogen bonding G 18-20, T 46 and $\mathrm{K} 126$ were involved in $\mathrm{PmRab7}$ activation. In the present molecular docking study, CID 1067700 was docked with PmRab7 (Target protein PDB Id: 1VG8) using the docking program GLIDE in the presence of $\mathrm{Mg}^{2+}$ and GTP. Total 70 compounds taken for docking, among them 10 were resulted best interactions with PmRab7 in XP glide docking, from that compounds were selected and subjected to induced fit docking. GTP and CID 106700 interaction showed the same GTP active site residues with high docking score $9.21 \mathrm{Kcal} / \mathrm{Mol}(\mathrm{GTP})$ and $7.89 \mathrm{Kcal} /$ Mol (CID-1067700) [24]. Ligplot reveal that structural and functional relationship depend on the hydrophobic interactions between $P m R a b 7$ amino acid residues with ring-fused pyran of CID1067700, moreover hydrogen bonding of carboxylic acid, extended $\mathrm{N}$-acyl thio urea linker and orientation of CID 1067700 also participating to functionally activate. Earlier Feng et al. [25] have identically reported that CID 1067700 inhibits nucleotide binding in wild type or mutant Rab7 in GTP and GDP bound states. These results show that PmRab7 GTPase inhibitor, binds to the nucleotide binding site on GTPases and utility of the CID 106700 also been studied on the GTPases for manipulating GTPase activities and tracking the consequences on known cellular pathways [26,27]. Rabs GTPase play essential role in molecule internalization and lysosomal degrading, hence inhibition of the Rab GTPases would be expected to delay their degradation. By the model structure analysis of PmRab7, Thr 22 amino acid residue shown interaction with $\mathrm{Mg}^{2+}$, which was responsible for guanine nucleotide binding likely to Agola et al. [28], eventually CID 1067700 also binding in the same binding packet of GTP after replacing GTP or $\mathrm{Mg}^{2+}$ also, this may suggest that $\mathrm{Mg}^{2+}$ is not essential for small molecule binding, Cray 
et al. [29] have also reveal in mutant Rab7T22N. Other than PmRab7 (Rab7) inhibition by CID 1067700 inhibitor, it has also been observed potential inhibitor against other small molecular weight GTPase likely Rab2, Rho-family GEF, Rac1 and Rho A [30]. By molecular docking it can predict that, CID 106700 is a competitive inhibitor which can nicely fit in nucleotide binding pockets of PmRab7 in both conformation GTP and GDP bound state. To validate the docking protocol, co-crystallized ligand was re-docked into the active site of PmRab7 complex. Cocrystal of GTP showed the hydrogen bond with GTP binding site residues like ALA156, SER34, ASP128, THR22, TYR37 and THR64, whereas co crystal of CID 1067700 replacing the $\mathrm{Mg}^{2+}$ and GTP from the complex and binding at same GTP binding site with other amino acid residues (SER34 and TYR37). The details mechanism of GTPase inhibitors has not been well studied yet, CID 106700 can inhibit guanine nucleotide binding to PmRab7 GTPases, the mechanism of inhibition of the CID 106700 towards Rab7 has been studied earlier, and it was demonstrated that CID 106700 can directly play role the guanine nucleotide binding in GTP or GDP. The previous study by Amrendra et al. [31] showed that, PmRab7 expressed 7-8-fold high in 72 hrs of infection when WSSV copy number was 82080 (Figures 7-9). Inhibiting function of wild type PmRab7 by selected small molecule inhibitor has shown partial blockage of WSSV gene expression in $P$. monodon up to certain period, suppressing the function of $P m R a b 7$ by double standard RNA can inhibit entry of WSSV and YHV (Yellow Head Virus) and knockdown PmRab7 by ds RNA has potential power to inhibit the WSSV infection in Laem-Singh virus by inhibiting WSSV replication. Whereas our remarkable result has shown small molecule can inhibit the internalization of virus up to a certain time of periods but may not have any role in WSSV replication.

\section{Conclusion}

A present study reveals $P m R a b 7$ structure as a highly organized, the domain of PmRab7 has one highly conserve region, five G box, two switch regions and one GTP binding site similar to all Rab7 monomeric from plant to animal, with one G box, GTP binding site and isoprenylation site. ALA156, SER34, ASP128, THR22, TYR37 and THR64 amino acid participating to bind with GTP GDP in $P m R a b 7$ pocket in presence of $\mathrm{Mg}^{++}$. SER34 and TYR37 are involved with CID 1067700 binding to PmRab7 by replacing $\mathrm{Mg}^{2+}$ in both conformations GTP and GDP bound state. Moreover, analyzing the binding mode and the knowledge of crucial interactions could provide insight for the design of new drugs for treating diseases associated with the reproductive, nervous, endocrine, immune, and cardiovascular systems, among others.

\section{Conflict of Interest}

All authors declared that they have no conflict of interest.

\section{Competing financial interests}

The authors declare no competing financial interests.

\section{References}

1. Colicelli J (2004) Human RAS superfamily proteins and related GTPases. Sci STKE 250: re13.

2. Parks WT, Frank DB, Huff C, Renfrew HC, Martin J, et al. (2001) Sorting nexin 6 , a novel SNX, interacts with the transforming growth factor- $\beta$ family of receptor serine-threonine kinases. J Biol Chem 276: 19332-19339.

3. Samantha LS, Cao C, Pylypenko O, Rak A, Wandinger-Ness A (2007) Rab GTPases at a glance. J Cell Sci 121: 246.

4. Sasaki T, Kikuchi A, Araki S, Hata Y, Isomura M, et al. (1990) Purification and characterization from bovine brain cytosol of a protein that inhibits the dissociation of GDP from and the subsequent binding of GTP to smg p25A, a Ras p21-like GTP-binding protein. J Biol Chem 265: 2333-2337.

5. Rak A, Pylypenko O, Durek T, Watzke A, Kushnir S, et al. (2003) Structure of Rab GDP-dissociation inhibitor in complex with prenylated YPT1 GTPase. Science 302: 646-650.

6. Rak A, Pylypenko O, Niculae A, Pyatkov K, Goody RS, et al. (2004) Structure of the Rab7: REP-1 complex: Insights into the mechanism of Rab prenylation and choroideremia disease. Cell 117: 749-760.

7. Ullrich Q, Stenmark H, Alexandrov K, Huber LA, Kaibuchi K, et al. (1993) Rab GDP dissociation inhibitor as a general regulator for the membrane association of rob proteins. J Biol Chem 268: 18143-18150.

8. Soldati T, Shapiro AD, Dirac SAB, Pfeffer SR (1994) Membrane targeting of the small GTPase Rab9 is accompanied by nucleotide exchange. Nature 369 : 76-78.

9. Seals DF, Eitzen G, Margolis N, Wickner WT, Price A (2000) An Ypt/Rab effecto complex containing the Sec1 homolog Vps33p is required for homotypic vacuole fusion. Proc Natl Acad Sci USA 97: 9402-9407.

10. Cantalupo G, Alifano P, Roberti V, Bruni CB, Bucci C (2001) Rab-interacting lysosomal protein (RILP): The Rab7 effector required for transport to lysosomes. J Embo 20: 683-693.

11. Mizuno K, Kitamura A, Sasaki T (2003) Rabring7, a novel Rab7 target protein with a RING finger motif. Mol Biol Cell 14: 3741-3752.

12. Dong C, Sanchez LE, Price RA (2004) Relationship of obesity to depression: A family-based study. Int J Obes 28: 780-795.

13. Johansson M, Lehto M, Tanhuanpaa K, Cover TL, Olkkonen VM (2005) The oxysterol-binding protein homologue ORP1L interacts with Rab7 and alters functional properties of late endocytic compartments. Mol Biol Cell 16: 5480-5492.

14. Rojas R, Van Vlijmen T, Mardones GA, Prabhu Y, Rojas AL, et al. (2008) Regulation of retromer recruitment to endosomes by sequential action of Rab5 and Rab7. J Cell Biol 183: 513-526.

15. Pan X, Eathiraj S, Munson M, Lambright DG (2006) TBC-domain GAPs for Rab GTPases accelerate GTP hydrolysis by a dual-finger mechanism. Nature 442: 303-306.

16. Adamo DP, Menegon A, Lo Nigro C, Grasso M, Gulisano M, et al. (1998) Mutations in GDI1 are responsible for X-linked non-specific mental retardation. Nature Genet 19: 134-139.

17. Hadano S, Hand CK, Osuga H, Yanagisawa Y, Otomo A, et al. (2001) A gen encoding a putative GTPase regulator is mutated in familial amyotrophic latera sclerosis 2. Nat Genet 29: 166-173.

18. Livak KJ, Schmittgen TD (2001) Analysis of relative gene expression data using realtime quantitative PCR and the 2- $\triangle \triangle \mathrm{CT}$. Methods 25: 402-408.

19. Tang KFJ, Lightner DV (2006) Infectious hypodermal and hematopoietic necrosis virus (IHHNV) in the genome of the black tiger prawn Penaeus monodon from Africa and Australia. Virus Res 118: 185-191.

20. Chalermporn O, Mayuree C, Kallaya S, Sakol P (2007) Suppression of PmRab7 by dsRNA inhibits WSSV or YHV infection in shrimp. Mar Biotechnol.

21. Amrendra K, Vaishnavi R, Lakshminarayanan K, Saravanakumar A (2014) Cloning, system analysis and model structural information of PmRab7 protein. Int J Curr Biotechnol 2: 1-6.

22. Kallaya S, Wanphen W, Flegel TW (2006) PmRab7 Is a VP28-binding protein involved in white spot syndrome virus infection in shrimp. J Virol 80: 10734 10742.

23. Arunima G, Andy TV, Beverly KK, Colin AK, Mary AM, et al. (2012) Characterization of Zebrafish von willebrand factor reveals conservation of domain structure, multimerization, and intracellular Storage. Adv Hemato Article ID: 214209

24. Ling H, Kenney SR, Phillips GK, Simpson D, Schroeder CE, et al. (2013) Characterization of a Cdc42 protein inhibitor and its use as a molecular probe. J Biol Chem 288: 8531-8543.

25. Feng Y, Press B, Wandinger NA (1995) Rab7: An important regulator of late endocytic membrane traffic. J Cell Biol 131: 1435-1452.

26. Moniz S, Matos P, Jordan P (2008) WNK2 modulates MEK1 activity through the Rho GTPase pathway. Cell Signal 20: 1762-1768. 
Citation: Kumar A, Shekhar S, Saravana kumar A (2017) Role of PmRab7 Regulation in WSSV Infection and Functional Validation of Small Molecule as PmRab7 GTPase Inhibitor. J Aquac Res Development 8: 511. doi: 10.4172/2155-9546.1000511

27. Keely PJ, Conklin MW, Gehler S, Ponik SM, Provenzano PP (2007) Investigating integrin regulation and signalling events in three-dimensional systems. Methods in Enzymology 426: 27-45.

28. Agola JO, Hong L, Surviladze Z, Ursu O, Waller A, et al. (2012) A competitive nucleotide binding inhibitor: In vitro characterization of Rab7 GTPase inhibition. ACS Chem Biol 7: 1095-1108.

29. McCray BA, Skordalakes E, Taylor JP (2010) Disease mutations in Rab7 result in unregulated nucleotide exchange and inappropriate activation. Human Mol
Genet 19: 103

30. Zhang X, Schaffitzel C, Ban N, Shan SO (2009) Multiple conformational switches in a GTPase complex control co-translational protein targeting. Proc Natl Acad Sci USA 106: 1754-1759.

31. Amrendra K, Vaishnavi R, Kiran DR, Saravanankumar A (2016) Revealing localization and regulation of GTPase PmRab7 in lymphoid cells of Penaeus monodon after WSSV infection. J Coastal Life Med 4: 846-851. 